\title{
PHOTOCONDUCTIVE INFRARED EXCITATION SPECTRUM OF GaP DIODE WAFERS AND APPLICATION AS AN INFRARED IMAGING SYSTEM
}

\author{
K. Moser*, M. Brechter, and W. Prettl \\ Institut für Angewandte Physik \\ Universität Regensburg \\ 8400 Regensburg, West Germany
}

Received August 13, 1987

The photoconductive excitation spectrum of GaP:N ( $Z$ nTe) diodes was measured irradiating the samples from $p-$ and $n-s i d e$ unter forward and reverse bias conditions. The ionization thresholds of $S$ and Te donors as well as transitions from their ground states to excited bound levels with subsequent ionization by the electric field could be seen. The observed minima in the continuum above the ionization threshold are due to phonon assisted fast recombination. The photoconductive signal by irradiation from the $p-s i d e$ is greater and sets on at lower photon energies. In addition, the transmission spectrum was also investigated and interpreted. The green photoluminescence resulting from the recombination of infrared generated carriers in the pn-junction can be applied for infrared imaging if GaP diode wafers of suitable size are employed.

\section{Introduction}

Extrinsic photoconductivity and infrared excited electroluminescence have been observed in GaP light emitting diodes (LEDs) /1/ providing a simple, fast $/ 2-5 /$, and sensitive detector in the mid infrared spectral range up to $20 \mathrm{~mm}$ wavelength /6/. At sufficiently low temperatures 
neutral shallow impurities are ionized by infrared photons of suitable quantum energy, and electrons and holes drift under the influence of an electric bias field to the pn-junction where they recombine radiatively emitting green 1 ight $/ 4,6 /$.

In this paper a detailed investigation of the infrared excitation spectrum of large planar GaP diodes is presented. The electrodes were prepared in form of metal meshes allowing irradiation through both the $n-$ and $p$-regions of the diodes. The photocurrent was determined for various forward and reverse bias voltages and will be compared to the transmission spectrum of the samples. In addition the potential application of large planar GaP diode wafers for infrared imaging is demonstrated making use of the infrared excited electroluminescence.

\section{Experimenta}

For all investigations reported in this paper GaP:N (Zn,Te) diode wafers with an area of $1 \mathrm{~cm}^{2}$ were used. These samples were prepared by liquid phase epitaxy on $n$-conducting substrates with a Te concentration of $10^{17} \mathrm{~cm}^{-3}$ and $250 \mu \mathrm{m}$ thickness. The first epitaxial layer was also n-doped by diffusion of Te atoms from the substrate and is $20 \mathrm{~mm}$ thick. It is followed by a $10 \mu \mathrm{m}$ layer containing $10^{19}$ atoms $\mathrm{cm}^{-3}$ nitrogen. The uppermost $20 \mathrm{~mm}$ are doped with $\mathrm{Zn}\left(5 \times 10^{18}-10^{19} \mathrm{~cm}^{-3}\right)$ to obtain the p-zone. The alloyed metal electrodes have the form of narrow grids and cover approximately $15 \mathrm{p} . \mathrm{c}$. of the surface; they consist of $A u-Z n$ on the $p-f a c e$, and $A u-G e$ on the $n$-face.

For the spectroscopic investigations these samples were mounted in a temperature variable cryostat contained in the infrared spectrometer described earlier /6/. The spectral range of the investigation was from 560 to $1260 \mathrm{~cm}^{-1}$ and al 1 measurements were carried out at $6 \mathrm{~K}$ temperature, if not stated otherwise, and at 17.5 to $35 \mathrm{~V}$ bias voltages. The reference and transmitted beams were detected with a Golay cell and all signals were 
monitored by standard lock-in technique across a $1 \mathrm{M} \Omega$ load resistor.

For infrared imaging the sample was mounted in an immersion cryostat ( $t$ emperature $T=1.6 \mathrm{~K}$ ) with large KRS-5 windows. The objects, electrically heated wires, were focussed into the wafer by a Znse lens with 1.5 inches focal length. The upconverted visible image was then photographed with a conventional camera through quartz windows. Through the KRS-5 windows only red and infrared light is transmitted, thus reducing visible background radiation. The objects, however, were not emitting visible light.

\section{Results and Discussion}

a) Infrared Excitation Spectra

Fig. 1 shows the photoconductivity spectrum of a sample irradiated on the p-side for various forward bias voltages. Identified transitions from Te- and S-donors are indicated by arrows. As already observed $/ 6 /$, the diodes contain a similar amount of $S$ and Te though they were intentionally doped with Te only. The spectral positions of all features are in good agreement with i iterature values $/ 7-9 /$.

Direct electron transitions from the donor ground state to the conduction band minimum can take place above the donor ionization energy, this is denoted by $1 s \rightarrow \Delta$. Above this energy the sensitivity of the sampte is greatly enhanced. At $887 \mathrm{~cm}^{-1}$ there is a peak due to the transition from the s-donor ground state to the conduction band at the $X$-point (see insert Fig. 1), denoted by $1 s \rightarrow X_{1}^{c}$. Below the $T e$ and $S$ ionization energies there are additional structures due to optically induced transitions from the donor ground states to their excited states. From these levels the electrons are then excited to the conduction band by thermal phonons and electric field ionization $/ 6 /$. The $1 s \rightarrow 3 p_{0}$ transition of $s$ can be seen much more clearly at $35 \mathrm{~V}$ bias 


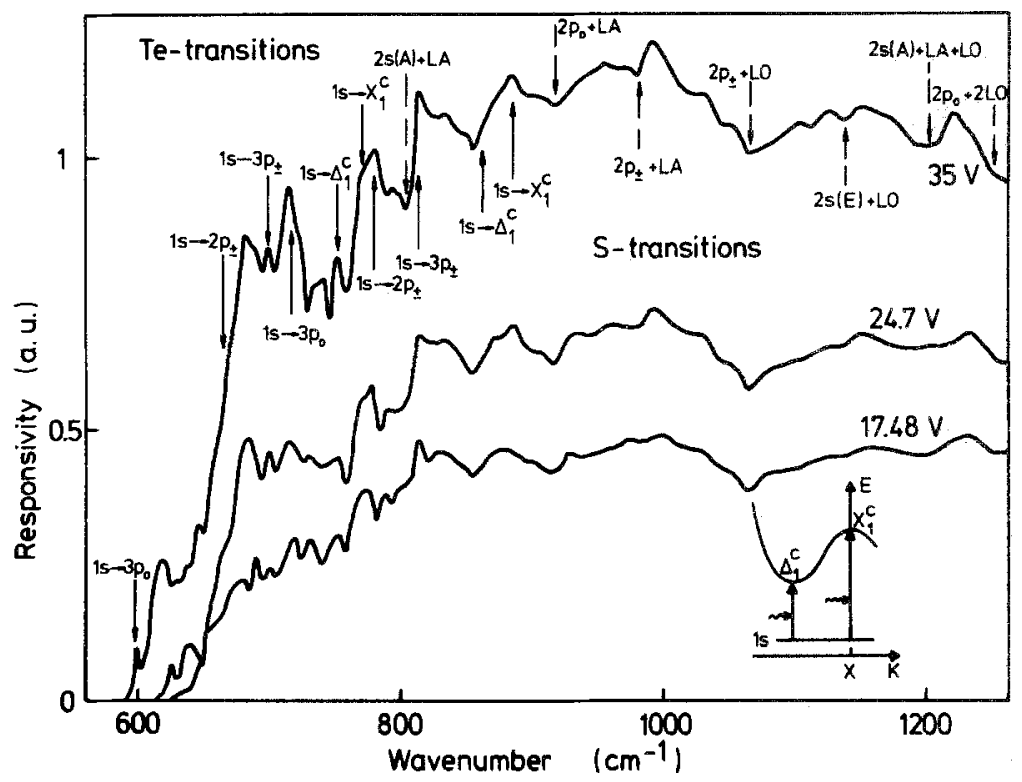

Figure 1. Spectral dependence of current responsivity of a GaP:N ( $Z n, T e)$ LED wafer at $6 \mathrm{~K}$ and for $17.5,24.7$ and $35 \mathrm{~V}$ forward bias voltage, irradiation on p-side. Impurity transitions are indicated by arrows (downward: Te-transitions, upward: S-transitions). Insert: IR induced transitions from the 1 s donor ground state to the $\Delta \frac{C}{1}$ and $x_{1}^{c}$ points of the conduction band.

voltage than at $17.5 \mathrm{~V}$. The situation is quite similar for the $1 \mathrm{~s} \rightarrow 2 \mathrm{p}_{+}$and $3 \mathrm{p}_{+}$transitions of the same impurity. The $1 s \rightarrow 3 p{ }^{ \pm}$transition of Te can, however, only be observed at $35 \mathrm{~V}$ bias voltages, not at 24.7 and $17.5 \mathrm{~V}$, this being a clear indication that the higher levels are ionized by the electric field. 
The photoionization of Zn acceptors whose threshold is expected to be at $561 \mathrm{~cm}^{-1}$ (corresponding to 69.7 meV) is not observed, which is due to the very short lifetime of holes because of fast free-to-bound donor recombinations $/ 4 /$. At 680 and $830 \mathrm{~cm}^{-1}$ there are structures $\checkmark$ isible that can be ascribed to deep donors or not yet identified donor complexes.

Above the donor ionization energies there are dips in the continuum that have been observed previously in bulk GaP by $W$. Scott $/ 9 /$, but could not be recognized in earlier investigations of sma11 GaP - LEDs /6/. These structures are caused by fast relaxation of electrons from the conduction band to excited bound donor states under emission of LO and LA phonons. Thus higher states are populated which cannot be reached directly from the ground state by optical transitions, e.g. $2 \mathrm{~s}(A)$; the transition being denoted by $2 s(A)+$ LO. These processes have been observed for both Te and $S$ donors, being strongest at $1064 \mathrm{~cm}^{-1}$ because there the transitions $2 \mathrm{P}_{ \pm}+$LO of $\mathrm{Te}$ and $2 \mathrm{~s}(\mathrm{~A})+\mathrm{LO}$ of s overiap.

Thus the different spectral structures below the donor photoionization threshold at various bias voltages can be understood by electric field ionization of excited states.

If the wafer is irradiated from the n-side the excitation spectra look similar in principle (see Fig. 2). The ionization thresholds $1 s \rightarrow \Delta C$ and $1 \mathrm{~s} \rightarrow X_{1}^{C}$ for $T e$ and $S$ as well as field ioni $\frac{1}{2}$ ed higher states and phonon assisted recombination are observed showing also the above described dependence on bias field.

There are, however, also differences to i rradiation from the p-side. The signal is smaller and the low frequency onset is at $670 \mathrm{~cm}^{-1}$, the largest signal value is obtained at the $1 s \rightarrow 3 p_{ \pm}$ transition of $S$. The reason for that can be found in the absorption of the n-substrate which is approximately $500 \mathrm{~cm}^{-1}$ as for a layer of $220 \mathrm{\mu m}$ an average transmission of $3 \times 10^{-5}$ can be found. 


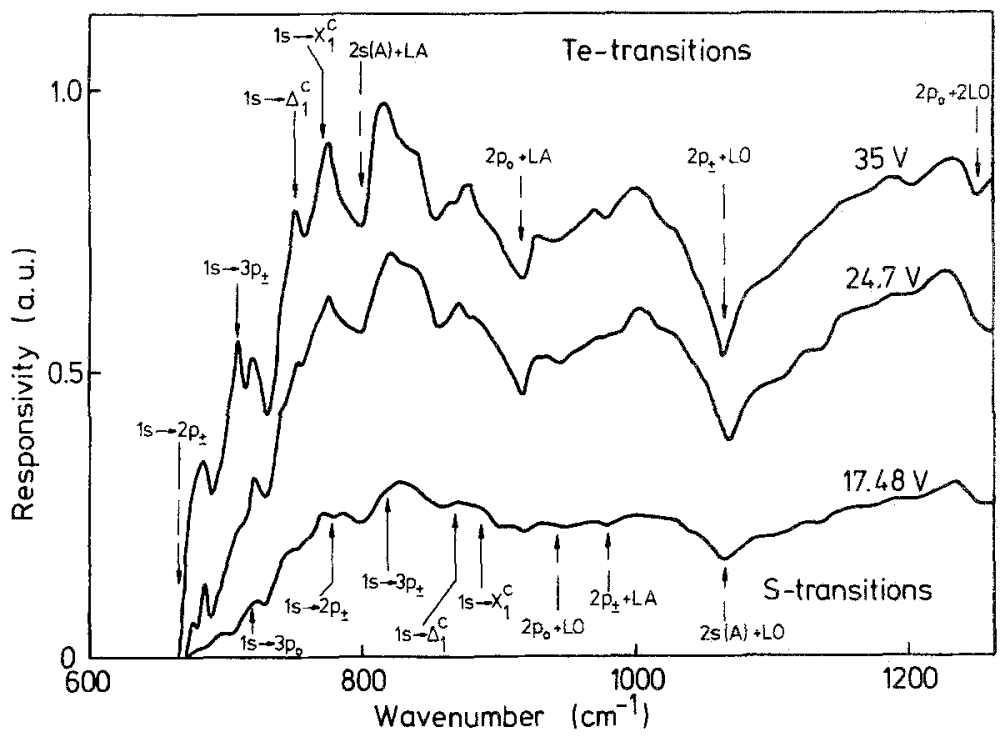

Figure 2. Like Fig. 1 but irradiation on $n-s i d e$.

Only when the sample is irradiated on the p-side ionization can take place close to the pn-junction where there are higher electric bias fields resulting in a higher photoconductive gain. Therefore when the sample is irradiated on the n-face also little field ionization takes place and the photocurrent can only be measured above $670 \mathrm{~cm}^{-1}$. The $1 \mathrm{~s} \rightarrow 3 p_{+}$transition of $s$ is strongest here because of the very strong absorption for this line (Fig. 4). So there is a very high population density of the $3 \mathrm{p}_{ \pm}$ state which is then ionized by the electric field; correspondingly the photoconductive signal increases very much with increasing bias voltage. This 1 ine is not so prominent if irradiation from the p-side takes place, probably because of the still unknown absorption of the $p-l a y e r$. 


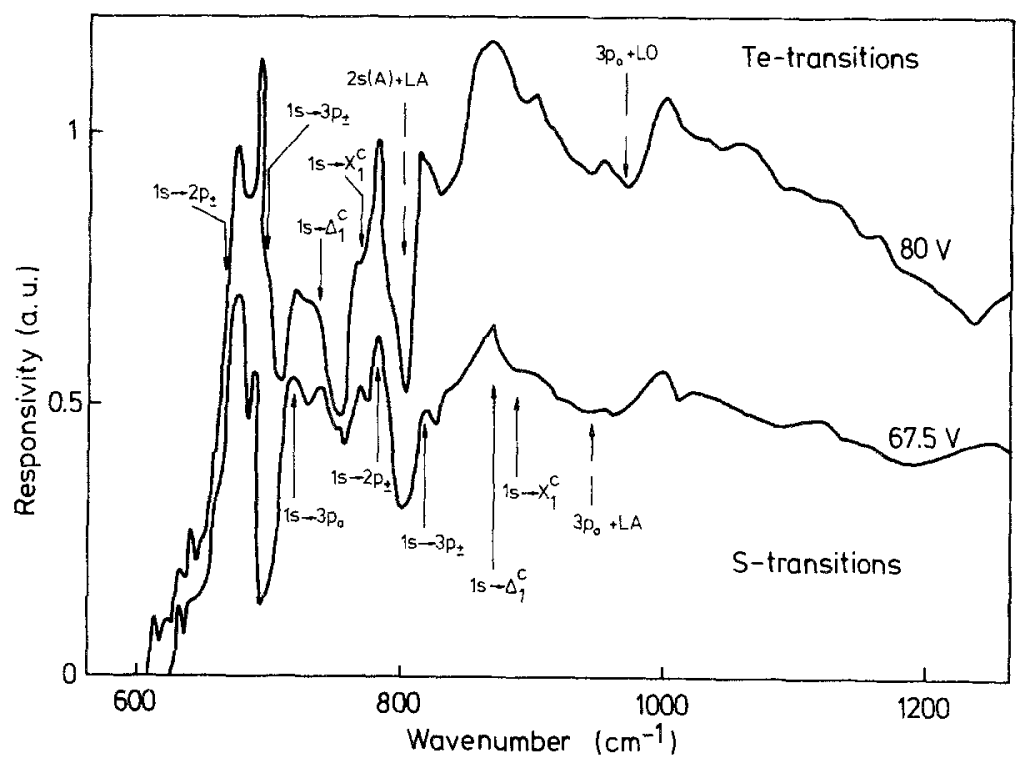

Figure 3. Spectral dependence of current responsivity of the GaP:N ( $Z n, T e)$ LED wafer at $6 \mathrm{~K}$ and for 67.5 and $80 \mathrm{~V}$ reverse bias voltage, irradiation on p-side. Impurity transitions are indicated by arrows (downward: Tetransitions, upward: S-transitions).

In reverse bias (see Fig. 3) we find substantial photocurrent and background current only above $50 \mathrm{~V}$ bias. The $\mathrm{s}-\mathrm{transitions} 1 \mathrm{~s} \rightarrow 3 \mathrm{p}_{\mathrm{p}}, 1 \mathrm{~s} \rightarrow 2 \mathrm{p}$, $1 s \rightarrow 3 p+$ are very distinctive. This is also true for the $S$ ionization $1 s \rightarrow \Delta C$, whereas $1 s \rightarrow X_{1}^{C}$ is only a weak shoulder beling due to the transmission behaviour. The excitations via Te are somewhat weaker but present: is $\rightarrow X_{1}^{C}, 1 s \rightarrow \Delta C$, and $1 s \rightarrow 2 p_{ \pm}$. The $1 s \rightarrow 3 p_{+}$transition can on t $^{\prime}$ be discerned above $75 \mathrm{~V}^{ \pm}$bias voltage as a weak shoulder of a structure near $690 \mathrm{~cm}^{-1}$ which could be ascribed to a deeper donor, but is most probably caused by the $1 s \rightarrow 2 p_{ \pm}$excitation changed 
by the Stark effect. Otherwise nothing of this transition except the border of those lines could be seen. Above the ionization energy, phonon assisted recombination is again present.

If the sample is irradiated on the n-side under reverse bias only a very weak signal can be detected. The Te transition $1 s \rightarrow x_{1}^{c}$ would be located at a minimum of the responsivity, similarly the $1 s \rightarrow \Delta c$ ionization. The s-donor excitations are more clearly observed but the signal can only be detected above $690 \mathrm{~cm}^{-1}$, whereas irradiation on the p-face yields a photocurrent already at $610 \mathrm{~cm}^{-1}$. The reason for that is again the strong absorption of the n-layer and also immediate sweep-out of charge carriers in the very broad depletion zone. For irradiation on the p-face this means that many donors are in the pn-junction and therefore the signal is greater than in forward bias at high voltages. Interband tunneling of charge carriers, however, is present in any case and causes strong noise under reverse bias conditions.

\section{b) Transmission Spectra}

The transmission spectrum of a $220 \mathrm{\mu m}$ thlck n-doped substrate can be seen in Fig. 4. The maximum transmission is there approximately as smal1 as $4 \times 10^{-5}$ yielding an absorption coefficient of several hundred per $\mathrm{cm}$. There is a strong minimum around $730 \mathrm{~cm}^{-1}$ being due to the $\mathrm{S}-$ transition $1 s \rightarrow 3 p_{o}$ and the Te-ionization $1 s \rightarrow \Delta c$. We see also that the transmission at the Stransition $1 s \rightarrow X_{1}^{C}$ is higher than that at $1 s \rightarrow \triangle C$.

\section{c) Infrared Imaging}

In our case up-conversion is caused by the ionization of shallow impurities by infrared light and recombination of the excited charge carriers near the pn-junction at nitrogen (NN) and donor acceptor (DA) pairs /2,6/ where they 


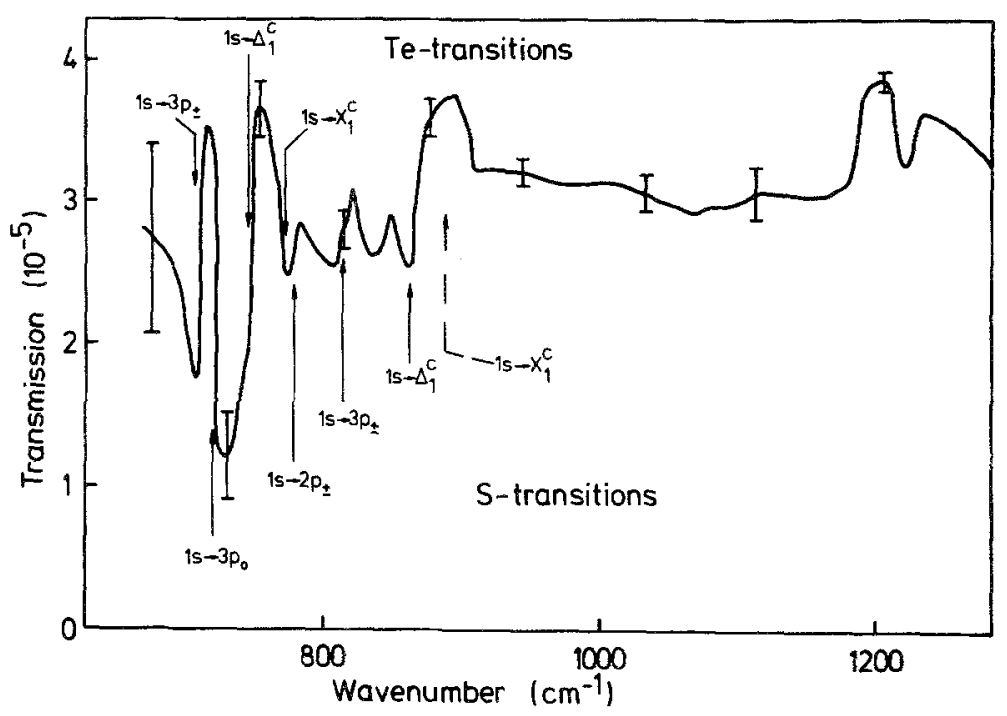

Figure 4. Transmission spectrum of a $220 \mathrm{~mm}$ thick Te-doped substrate at $6 \mathrm{~K}$. Impurity transitions are indicated by arrows (downward: Te-transitions, upward: S-transitions).

drifted under the influence of an external bias field. So it is possible to carry out infrared imaging if a sufficiently large wafer and an imaging optics (here a ZnSe lens) is used.

The objects taken in this experiment were electrically heated wires projected onto the p-side of the wafer. The temperature of the wires was low enough so that no glowing was visible by the naked eye. Best results concerning contrast and brightness could be obtained at $U_{\text {LED }}=25$ to $30 \mathrm{~V}$. Fig. 5 shows a bent wire and the infrared up-converted visible image photographed from the wafer at $30 \mathrm{~V}$ bias. The up-converted light is 

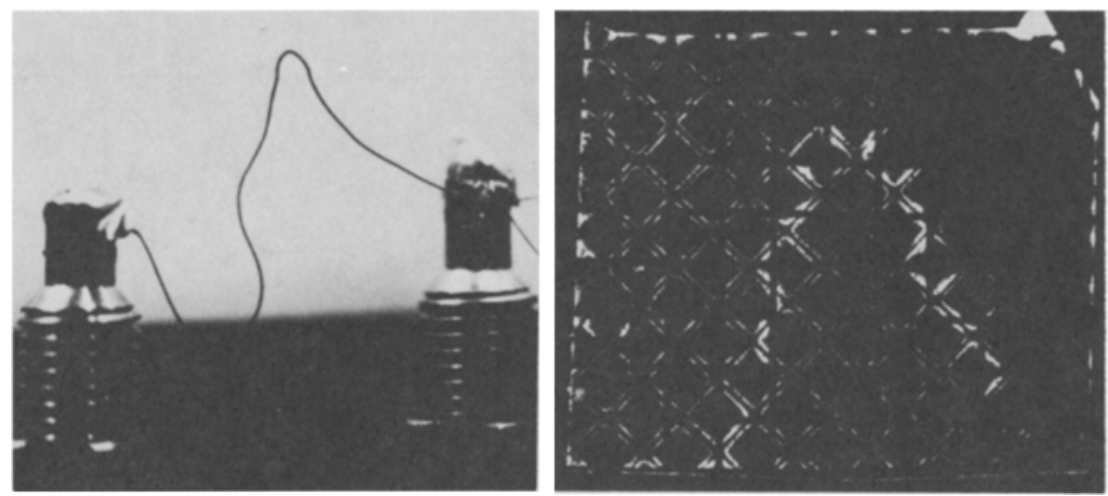

Figure 5 .

Left: Electrically heated wire as an object for infrared imaging.

Right: Infrared up-converted visible image of the wire as photographed from the Ga:N (Zn, Te) LED wafer with a conventional camera. Diode blas voltage $U_{\text {LED }}=30 \mathrm{~V}$, wafer temperature $T=1.6 \mathrm{~K}$.

generated preferably near the grid of electrodes where the electric field is largest. Thus better resolution may be obtained by using finer grids.

\section{Conclusion}

In this work photoconductive excitation spectra of GaP:N ( $Z n, T e)$ diode wafers were investigated. We found that irradiation on the p-side principally yields, a greater photocurrent and the signal can be seen over a lager spectral range than for irradiation on the $n-f a c e$. In the latter case some of the photoexcited higher levels cannot be ionized by the electric field.

Especially in reverse bias $p-s i d e$ irradiation yields a very much larger signal than n-side excitation. The main reason is that the infrared 
light is strongly absorbed in the n-substrate where the excited carriers do not very much vontribute to the photocurrent because of the lower electric field. If the sample however is irradiated from the p-side, the excited carriers are already quite close to the pn-junction. Under reverse bias higher voltages must be applied leading to higher electric fields in the pn-junction which can, however, have no effect on carriers generated near the $n$-face.

The main spectral features are the same in any case: direct ionization thresholds, excitation from ground states to bound excited levels with subsequent field ionization and phonon assisted recombination to bound states for $S$. and Te-donors yielding dips in the continuum.

The infrared up-converted visible light makes the construction of an infrared camera possible. So simple objects like heated wires can be made visible for the naked eye or an ordinary camera. This imaging system can, however, be improved by using better suited larger diode wafers with finer electrode grids.

\section{Acknowledgements}

We are indebted to Dr. N. Stath and J. Attenhauser (Siemens $A G$, Regensburg) for providing the diode samples and helpful discussions. We also thank $W$. Eisfeld for experimental support and $A$. Humbs für technical assistance. Financial support by the Deutsche Forschungsgemeinschaft is gratefully acknowledged. 


\section{References}

* Permanent address: Institut für Angewandte Physik, Universität Münster, 4400 Münster, W. Germany

/1/W. Eisfeld, U. Werling, and W. Prettl, App 1. Phys.Lett. 47,276 (1983).

/2/ K. Moser, W. Eisfeld, U. Werling, S. Wahl, and W. Prett1, Appl.Phys.Lett. 45, 711 (1984).

/3/ K. Moser, W. Eisfeld, and W. Prett 1 , Infrared Physics 4 , 659 (1985).

$14 /$ K. Moser, W. Eisfeld, W. Penzenstadler, and W. Prett1, J.Phys. D 18, 2303 (1985).

/5/ K. Moser and W. Prett 1 , Intern. J. of Infrared and Millimeter Waves I, 147 (1985).

/6/ K. Moser, S. Wahl, W. Eisfeld, and W. Prett, J.App 1.Phys. 57, $5438(1985)$.

/7/ A.A. Kopylov, A.N. Pikhtin, Solid State Commun. 26, 735 (1977).

/8/ Landolt-Börnstein, Zahlenwerte aus Naturwissenschaft und Technik, Gruppe III, Vol. 17, Springer Verlag Heidelberg 1982, p. $185 \mathrm{ff}$.

/9/W. Scott, J.Appl.Phys. 50, 472 (1979). 